\title{
Impacto del momento del diagnóstico en la evolución posoperatoria de los recién nacidos con cardiopatía congénita en un hospital público, en la Argentina
}

\author{
Impact of the time of diagnosis on the postoperative outcome of newborn infants \\ with congenital heart disease in a public hospital in Argentina
}

\author{
Dra. Mariela Krynski ${ }^{a}$ Dra. Mercedes Montonati ${ }^{a}$ y Dra. María Althabe ${ }^{a}$
}

\begin{abstract}
RESUMEN
Las cardiopatías congénitas representan el 13\% de la mortalidad infantil y el diagnóstico tardío aumenta la morbimortalidad. El objetivo del trabajo fue evaluar el impacto del momento del diagnóstico de los neonatos en la evolución posoperatoria. El momento del diagnóstico se clasificó en prenatal, anterior o posterior al alta de la maternidad. Se incluyeron 299 pacientes, con edad gestacional de $38 \pm 2,6$ semanas, peso de 3,22 $\pm 0,6$ $\mathrm{kg}$. Se operaron $266 ; 13$ fueron desestimados por características de la lesión; 10, por descompensación hemodinámica; y 10 fueron resueltos en Hemodinamia $u$ operados fuera del período neonatal. Solo 19 pacientes $(7 \%)$ tuvieron diagnóstico prenatal; en su mayoría, pacientes con obra social, residentes en Ciudad Autónoma de Buenos Aires o capitales de provincias. El momento del diagnóstico no se asoció con diferencias en la mortalidad; sí, en cambio, la mala condición clínica prequirúrgica, por la que falleció $3,6 \%$ de los pacientes antes del procedimiento. La supervivencia posquirúrgica fue $89,5 \%$; la global, $83 \%$.

Palabras clave: cardiopatía congénita, cirugía cardíaca, diagnóstico prenatal.
\end{abstract}

http:/ /dx.doi.org/10.5546/aap.2015.433

a. Unidad de Cuidados Intensivos 35 - Recuperación Cardiovascular. Hospital de Pediatría "Prof. Dr. Juan P. Garrahan". Ciudad Autónoma de Buenos Aires, Argentina.

Correspondencia:

Dra. Mariela Krynski: marikrynski@hotmail.com

Financiamiento: Ninguno.

Conflicto de intereses: Ninguno que declarar.

Recibido: 16-12-2014

Aceptado: 4-5-2015

\section{INTRODUCCIÓN}

Las cardiopatías congénitas (CC) son la primera causa de mortalidad en los pacientes con malformaciones congénitas. Afectan de 8 a 12 cada 1000 recién nacidos vivos y son una de las malformaciones congénitas más frecuentes y potencialmente graves. En nuestro país, representan el $13 \%$ de la mortalidad infantil, en tercer lugar luego de la prematurez y las infecciones. ${ }^{1}$ El diagnóstico tardío, en muchos casos, resulta en una elevada morbimortalidad perioperatoria. En este sentido, se han desarrollado diferentes estrategias para mejorar el diagnóstico de las cardiopatías congénitas. Entre ellas, el diagnóstico prenatal, que permite programar el nacimiento cerca o en un centro especializado y anticipar el soporte específico que necesitará el niño al nacer, como prostaglandinas, septostomía o cirugía de urgencia. La oximetría de pulso de rutina en los recién nacidos, como estrategia de pesquisa, puede resultar útil para mejorar la oportunidad diagnóstica de estos pacientes. ${ }^{2,3}$ En nuestro medio, no hay estudios que aporten información sobre el momento diagnóstico y su potencial repercusión en el tratamiento. El objetivo de este trabajo es describir las características de los recién nacidos con CC que ingresan a la Unidad de Cuidados Intensivos del Hospital Garrahan (UCI 35) y evaluar el impacto del momento del diagnóstico en su evolución posoperatoria.

\section{PACIENTES Y MÉTODOS}

Se incluyeron todos los pacientes menores de 60 días que ingresaron en la UCI 35 con diagnóstico de CC de resolución quirúrgica en el período neonatal, entre 2007 y 2011. Se registraron datos demográficos: edad al momento del diagnóstico, sexo, peso, lugar de residencia, control del embarazo, obra social, antecedentes familiares de cardiopatía. Datos del 
diagnóstico: momento del diagnóstico, tipo de cardiopatía, síntoma principal, condición clínica y concordancia diagnóstica. De la cirugía y del posoperatorio: tipo de reparación, mortalidad ajustada por riesgo con puntaje de RACHS-1. Se tomaron como límite de edad los 60 días para incluir a pacientes con diagnóstico tardío de CC de resolución habitual en el período neonatal. El momento diagnóstico se clasificó en prenatal, antes del alta y posterior al alta de la maternidad. Los síntomas se registraron como cianosis, shock, soplo e insuficiencia cardíaca. Se consideró mala condición clínica cuando el paciente presentaba requerimientos de asistencia respiratoria mecánica (ARM) y/o inotrópicos intravenosos (IV) al ingresar a la Unidad. Se consideró que existía concordancia cuando el diagnóstico de derivación coincidía con el defecto principal del paciente, aun cuando se hubieran identificado defectos asociados menores. El tipo de reparación quirúrgica se agrupó por complejidad utilizando la escala de RACHS-1, que consiste en seis categorías de riesgo de mortalidad creciente de acuerdo con la complejidad del procedimiento quirúrgico. ${ }^{4}$ Se realizó un análisis estadístico descriptivo; los datos se reportaron como media y DE o porcentaje, según correspondiera. Se exploraron como factores de riesgo de mortalidad el momento del diagnóstico y la condición clínica al ingresar (Stata 9.0). El protocolo fue aprobado por el Comité de Investigación del Hospital ( $\left.n^{\circ} 849 / 2014\right)$. Por tratarse de un estudio retrospectivo sin intervención, no se consideró necesario solicitar consentimiento informado.

\section{RESULTADOS}

Se incluyeron 299 pacientes, con una edad gestacional promedio de $38 \pm 2,6$ semanas y un peso de nacimiento de 3,22 $\pm 0,6 \mathrm{~kg}$. Del total de la población, se operaron 266 pacientes; 13 fueron desestimados por características de la lesión o malformaciones graves asociadas (12 de ellas, con ventrículo único); 10 no fueron intervenidos por descompensación hemodinámica; y 10 fueron resueltos en el Servicio de Hemodinamia u operados fuera del período neonatal (Figura 1). El $11 \%$ de la población analizada carecía de controles completos durante el embarazo; el $63 \%$ nació por parto vaginal; solo el 2,3\% tenía antecedentes de hermanos portadores de cardiopatía congénita; $19 \%$ contaba con obra social. El $47 \%$ de los pacientes provenían de la provincia de Buenos Aires; el 53\%, del interior; y el 7\% restante, de Ciudad Autónoma de Buenos Aires (CABA). Solo 19 pacientes $(7 \%)$ tuvieron diagnóstico prenatal, $65 \%$ durante la internación y $28 \%$ al momento del alta de la maternidad. En los casos de diagnóstico prenatal, 10 pacientes tenían diferentes formas de ventrículo único (Tabla 1). Los pacientes con obra social tuvieron mayor probabilidad de diagnóstico prenatal $(12,5 \%$ vs. $5,1 \%, p=0,047)$. De la misma manera, los que residían en la CABA o en las capitales de sus provincias tuvieron mayor probabilidad de diagnóstico prenatal que los que vivían en el interior $(8,7 \%$ vs. $1,2 \%, p=0,019)$. La forma de presentación más frecuentemente detectada fue la cianosis en el $68 \%$ de los casos, independientemente del momento del diagnóstico, seguida del

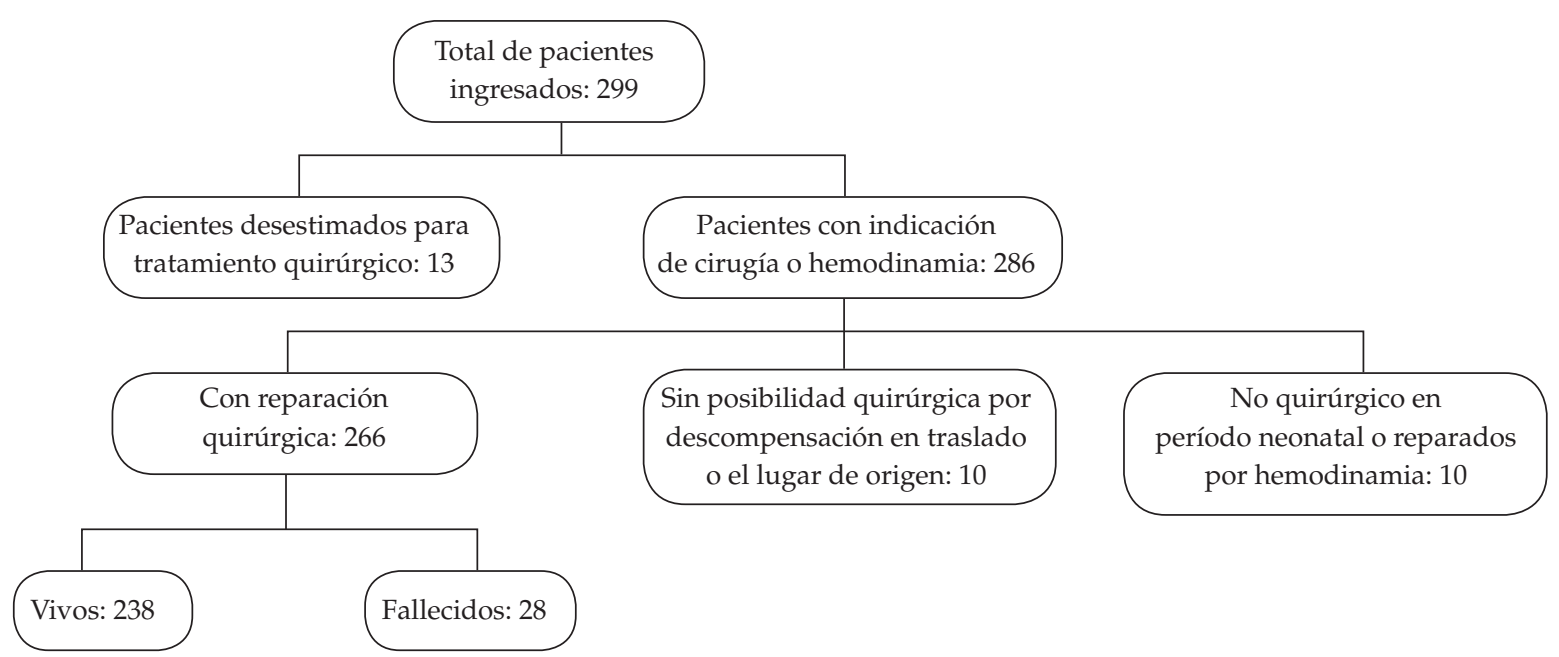


soplo en el $15 \%$, shock en el $9 \%$, insuficiencia cardíaca congestiva (ICC) en el 8\%. El 53\% de los pacientes se encontraban con ARM y con soportes inotrópicos IV y/o prostaglandinas al ingresar a la Unidad. Tomando toda la población, el tipo de CC más frecuente fue la trasposición de grandes arterias (TGA) simples y complejas; los restantes diagnósticos se describen en la Tabla 2. A todos los pacientes se les realizó un ecocardiograma doppler color al ingresar para confirmar el diagnóstico. La concordancia con el diagnóstico del centro de derivación fue $78 \%$. Setenta y ocho pacientes (26\%) requirieron cateterismo y/o tomografía axial computada (TAC) como estudios adicionales. Del total de pacientes con indicación de intervención (266), el 3,4\% perdieron la

TABLA 1. Malformaciones con diagnóstico prenatal

\begin{tabular}{lc}
\hline Patología & Número \\
\hline Hipoplasia de VI & 9 \\
Atresia pulmonar con CIV & 3 \\
Anomalía total del retorno venoso & 2 \\
CIV con coartación de aorta & 2 \\
Trasposición de grandes arterias & 1 \\
Ventrículo único no hipoplasia de VI & 1 \\
Tronco arterioso & 1 \\
Total & $\mathbf{1 9}$ \\
\hline
\end{tabular}

CIV: comunicación interventricular; VI: ventrículo izquierdo. posibilidad quirúrgica por mala condición clínica y fallecieron, la mayoría de ellos con posibilidad de buena reparación anatómica (3 trasposiciones de grandes vasos -TGV-, 2 interrupciones de arco aórtico con comunicación interventricular -CIV-, 1 tronco arterioso). La complejidad de las cirugías fue alta de acuerdo con la escala de RACHS-1, con más de 70\% de los casos en categoría $\geq 3$ (R1: 1\%; R2: 15\%; R3: 40\%; R4: 31\%; y R5/6: 13\%). La mortalidad posquirúrgica fue de $10,5 \%$, mientras que la mortalidad global de la serie fue $17 \%$. En el análisis univariado, el momento del diagnóstico no se asoció de manera significativa con diferencias en la mortalidad (18\% los prenatales vs. $9,7 \%$ los posnatales, $\mathrm{p}=0,25)$. Sin embargo, los pacientes con mala condición previa tuvieron una mortalidad más elevada ( $22 \%$ vs. $9 \%, \mathrm{p}=0,003)$.

\section{DISCUSIÓN}

Los progresos en cirugía cardiovascular neonatal, técnicas quirúrgicas, de anestesia y perfusión han permitido mejoras importantes en la supervivencia de pacientes con malformaciones muy complejas. El diagnóstico temprano constituye uno de los pilares en el tratamiento oportuno de estos pacientes, ya que permite una adecuada estabilización preoperatoria, una apropiada coordinación para el traslado y mejores condiciones preoperatorias. ${ }^{5}$ Como observamos en nuestra serie, los pacientes con

TABla 2. Diagnóstico en orden de frecuencia

\begin{tabular}{lcc}
\hline Cardiopatía congénita & Frecuencia absoluta & Frecuencia relativa (\%) \\
\hline TGV & 82 & 27,4 \\
HVI & 41 & 13,7 \\
ATRV & 40 & 13,4 \\
VU no HVI & 28 & 9,3 \\
CIV + CoAo & 18 & 6,0 \\
AP c/CIV & 13 & 4,3 \\
IAA & 13 & 4,3 \\
CoAo & 12 & 4,0 \\
Tronco & 10 & 3,3 \\
CIV & 7 & 2,3 \\
Fallot & 4 & 1,3 \\
Shone & 2 & 0,6 \\
Canal auriculoventricular & 2 & 0,6 \\
Ductus & 1 & 0,3 \\
Otros & 26 & 8,7 \\
Total & 299 & \\
\hline
\end{tabular}

TGV: trasposición de grandes vasos; HVI: hipoplasia de ventrículo izquierdo; ATRV: anomalía total del retorno venoso; VU no HVI: ventrículo único no hipoplasia de ventrículo izquierdo; CIV + CoAo: comunicación interventricular + coartación de aorta; AP c/CIV: atresia pulmonar con comunicación interventricular; IAA: interrupción del arco aórtico. 
mala condición preoperatoria tienen peores resultados quirúrgicos y hasta pueden perder la oportunidad de una cirugía reparadora. ${ }^{6}$ Si bien el diagnóstico ha mejorado en los últimos años, muchos pacientes continúan diagnosticándose luego del alta y el porcentaje de pacientes con diagnóstico prenatal es muy reducido. Los pacientes que nacieron en CABA o provincia de Buenos Aires y/o aquellos que poseían obra social tuvieron mayor posibilidad de diagnóstico prenatal, lo que pone en evidencia algunas desigualdades del sistema. Este dato es más llamativo por tratarse de una población seleccionada, ya que el Hospital Garrahan es el centro de derivación de mayor volumen y complejidad en cirugía cardiovascular pediátrica en el país; en él se operan 60 recién nacidos por año con circulación extracorpórea. En la literatura, centros de similares características describen un 30-50\% de pacientes de este tipo con diagnóstico prenatal. ${ }^{7,8} \mathrm{El}$ diagnóstico prenatal por sí solo no se relaciona con menor mortalidad posoperatoria, ya que, en este resultado, interviene una enorme cantidad de variables, entre ellas, la gran variedad de patologías y procedimientos quirúrgicos. Sin embargo, es de gran importancia para reducir la mortalidad por cardiopatía congénita en un país extenso, con pocos centros capacitados para la resolución de casos complejos y con dificultades en procesos de derivación y traslado como el nuestro. ${ }^{9}$

La mortalidad posoperatoria en cirugía cardíaca neonatal en su conjunto fue $10,5 \%$, mientras que la mortalidad de estas patologías sin cirugía alcanzó el 90\%. Es claro, entonces, que la reducción de la mortalidad en las cardiopatías congénitas se logra a través de la cirugía reparadora o paliativa, toda vez que las características de la malformación lo permitan. En nuestra serie, si se hubieran intervenido los 10 pacientes que perdieron la posibilidad por su condición, la mortalidad global podría haber disminuido en 3 puntos porcentuales, de 17\% a $14 \%$. La demora en el diagnóstico posnatal reconoce múltiples causas: falta de sospecha, falta de profesionales capacitados o puede estar vinculada a pacientes con menor sintomatología. Pueden ser pacientes con formas menos graves o cuya sintomatología está vinculada con las modificaciones hemodinámicas que se producen en el período neonatal, como el cierre del ductus o la reducción de las resistencias pulmonares. El diseño de este estudio no nos permite avanzar en conclusiones en este sentido, como tampoco realizar estimaciones de qué número de pacientes con malformaciones graves pueden haber fallecido sin diagnóstico de CC.

Por estas razones, creemos que, en ausencia de diagnóstico prenatal, la pesquisa con oximetría de pulso previo al momento del alta podría ser una estrategia útil para mejorar la oportunidad diagnóstica y disminuir la morbimortalidad en las cardiopatías congénitas. ${ }^{2}$ Si bien, en este estudio, no se evaluaron resultados en otras áreas de impacto a largo plazo, como el desarrollo neurológico, la literatura hace hincapié en intentar disminuir la morbilidad de presentación temprana, como asfixia y convulsiones prequirúrgicas, con repercusión a largo plazo en el neurodesarrollo y en las cardiopatías que se presentan como shock en las primeros días de vida. ${ }^{10-12} \mathrm{La}$ concordancia diagnóstica con el centro derivante puede considerarse razonable, teniendo en cuenta que, en el $26 \%$ de los casos, se requirieron estudios adicionales, lo que habla de la complejidad de este grupo de patologías. Esta información es relevante a la hora de definir el nivel de capacitación que este tipo de diagnósticos requiere.

\section{CONCLUSIONES}

El diagnóstico de cardiopatía congénita fue prenatal en el $7 \%$ de los casos y, en $28 \%$, se realizó con posterioridad al alta. Vivir en la región metropolitana y/o poseer obra social se asoció con mayor posibilidad de diagnóstico prenatal. El momento diagnóstico no se asoció con mayor mortalidad posoperatoria; sin embargo, 3,6\% de los pacientes con indicación de reparación falleció antes del procedimiento por mala condición clínica. La supervivencia de los pacientes intervenidos fue $89,5 \%$.

\section{REFERENCIAS}

1. Argentina. Ministerio de Salud. Dirección de Estadísticas e Información en Salud. Estadísticas vitales. Información básica año 2012. Buenos Aires, 2013. [Acceso: 10 de octubre de 2014]. Disponible en: http://www.deis.gov. ar/publicaciones/archivos/Serie5Nro56.pdf.

2. Mahle WT, Martin GR, Beekman RH 3rd, Morrow WR. Endorsement of Health and Human Services recommendation for pulse oximetry screening for critical congenital heart disease. Pediatrics 2012;129(1):190-2.

3. Peterson C, Ailes E, Riehle-Colarusso T, Oster ME, et al. Late detection of critical congenital heart disease among US infants: estimation of the potential impact of proposed universal screening using pulse oximetry. JAMA Pediatr 2014;168(4):361-70.

4. Jenkins KJ, Gauvreau K, Newburger JW, Spray TL, et al. Consensus-based method for risk adjustment for surgery 
for congenital heart disease. J Thorac Cardiovasc Surg 2002;123(1):110-8.

5. Dawson AL, Cassell CH, Riehle-Colarusso T, Grosse SD, et al. Factors associated withlate detection of critical congenital heart disease in newborns. Pediatrics 2013;132(3):e604-11.

6. Chang RK, Gurvitz M, Rodriguez S. Missed diagnosis of critical congenital heart disease. Arch Pediatr Adolesc Med 2008;162(10):969-74.

7. Fuchs IB, Müller H, Abdul-Khaliq H, Harder T, et al. Immediate and long-term outcomes in children with prenatal diagnosis of selected isolated congenital heart defects. Ultrasound Obstet Gynecol 2007;29(1):38-43.

8. Brown KL, RidoutDA, Hoskote A, Verhulst L, et al. Delayed diagnosis of congenital heart disease worsens preoperative condition and outcome of surgery in neonates. Heart 2006;92(9):1298-302.

9. SatomiG, YasukochiS, Shimizu T, TakigikuK, etal. Has fetal echocardiography improved the prognosis of congenital heart disease? Comparison of patients with hypoplastic left heart syndrome with and without prenatal diagnosis. Pediatr Int 1999;41(6):728-32.

10. Mahle WT, Clancy RR, McGaurn SP, Goin JE, et al. Impact of prenatal diagnosis on survival and early neurologic morbidity in neonates with the hypoplastic left heart syndrome. Pediatrics 2001;107(6):1277-82.

11. Bartlett JM, Wypij D, Bellinger DC, Rappaport LA, et al. Effect of prenatal diagnosis on outcomes in D-transposition of the great arteries. Pediatrics 2004;113(4):e335-40.

12. Kumar RK, Newburger JW, Gauvreau K, Kamenir SA, et al. Comparison of outcome when hypoplastic left heart syndrome and transposition of the great arteries are diagnosed prenatally versus when diagnosis of these two conditions is made only postnatally. Am J Cardiol 1999;83(12):1649-53.

\title{
Implementación de un programa para prevenir el daño neurológico de la encefalopatía hipóxico isquémica en Buenos Aires. Hipotermia terapéutica
}

\author{
Implementation in Buenos Aires City of a program to prevent neurological \\ damage caused by hypoxic-ischemic encephalopathy. Therapeutic hypothermia
}

\author{
Dra. Mariana Valera ${ }^{a}$, Dr. Juan Pablo Berazategui ${ }^{a}$ Dra. Gladys Saa ${ }^{a}$, Dra. Carolina Olmo Herrera ${ }^{a}$, \\ Dra. Teresa Sepúlveda ${ }^{a}$,Dra. María Fernanda Buraschi ${ }^{a}$, Dr. Sebastián Gacio ${ }^{b}$, Lic. Cristina Villalba ${ }^{a}$, \\ Lic. Inés Belosoc, Dra. Graciela Basso ${ }^{a}$, Dr. Waldemar Carlo y Dr. Jorge Tavosnanska ${ }^{a}$
}

a. Unidad de Neonatología.

b. Servicio de Pediatría.

c. Servicio de Fonoaudiología.

Hospital General de Agudos "Juan A. Fernández"

(HGAJAF), Ciudad Autónoma de Buenos Aires,

República Argentina.

d. División de Neonatología, Universidad de Alabama, EE.UU.

\section{Correspondencia:}

Dr. Jorge Tavosnanska, jtavos@intramed.net

Financiamiento: Ninguno.

Conflicto de intereses: El Servicio de Neonatología del Hospital General de Agudos "Juan A. Fernández" recibió en calidad de préstamo los equipos Cool Cap ${ }^{\circledR}$ de la empresa Medix ${ }^{\circledR}$ by Natus ${ }^{\circledR}$ para hipotermia selectiva y Amrra-Term ${ }^{\circledR}$ para hipotermia corporal total, además del equipo CFM Olympic $6000 \AA$ de la empresa Medix $®$ by Natus $₫$ para el monitoreo de la función cerebral.

\section{RESUMEN}

La hipotermia terapéutica es el estándar de cuidado para la encefalopatía hipóxica isquémica (EHI). Se regionalizó este tratamiento en la red perinatal de la Ciudad de Buenos Aires. Los objetivos de esta presentación son los siguientes: 1. Describir la implementación del programa de hipotermia en la red; 2. Reportar la mortalidad, complicaciones y efectos adversos asociados al tratamiento.

El programa se implementó en etapas: 1) 2009-2010. Capacitación y entrenamiento en el uso del equipamiento. 2) 2010-2014. Tratamiento y seguimiento de los pacientes con EHI moderada o grave. Hasta octubre de 2014, 27 neonatos recibieron hipotermia terapéutica con EHI moderada $(n=15)$ y grave $(n=12)$. Ninguno falleció durante el tratamiento. Se perdieron 3 neonatos en el seguimiento. Delos 16 sobrevivientes mayores de un año, 3 tienen discapacidad neurológica grave. Fue factible implementar el programa. Es prioritario capacitar para la identificación de pacientes con EHI.

Palabras clave: recién nacido, encefalopatía hipóxica isquémica, hipotermia terapéutica.

http:/ /dx.doi.org/10.5546/aap.2015.437 